Pure Appl. Chem., Vol. 84, No. 10, pp. 2045-2054, 2012.

http://dx.doi.org/10.1351/PAC-CON-11-09-23

(C) 2012 IUPAC, Publication date (Web): 8 April 2012

\title{
Pulsed chronopotentiometric membrane electrodes based on plasticized poly(vinyl chloride) with covalently bound ferrocene functionalities as solid contact transducer*
}

\author{
Marcin Pawlak, Ewa Grygolowicz-Pawlak, and Eric Bakker ${ }^{\ddagger}$ \\ Department of Inorganic, Analytical and Applied Chemistry, University of Geneva, \\ Quai E.-Ansermet 30, CH-1211 Geneva, Switzerland
}

\begin{abstract}
Ion-selective membrane materials based on poly(vinyl chloride) (PVC)-containing covalently attached redox-active ferrocene $(\mathrm{Fc})$ groups are characterized here as all-solidstate pulsed voltammetric ion sensors. The redox capacity of the membrane increases 7-fold with a doubling of the $\mathrm{Fc}$ content and 3-fold with the addition of $10 \mathrm{wt} \%$ of the lipophilic electrolyte ETH 500, tetradodecylammonium tetrakis(4-chlorophenyl)borate. This salt improves the ionic conductivity of the membrane and appears to make the Fc groups electrochemically more accessible. A too high content of the two, on the other hand, was found to cause undesired sensitivity to redox-active species present in the sample solution. Dilution of the membrane with a plasticizer eliminated this redox sensitivity while preserving its high redox capacity. A practical application of the designed electrodes in electrochemical analysis was demonstrated with a multi-pulse protocol that includes a current-controlled ion uptake pulse, followed by an open-circuit potential (OCP) measurement and a regeneration pulse. Potentiometric calibration curves obtained with this protocol exhibited a linear response with near-Nernstian slopes for acetate, nitrate, chloride, and perchlorate ions with the selectivity expected for an ion-exchanging membrane.
\end{abstract}

Keywords: analytical chemistry; click chemistry; electrochemistry; ion-selective electrodes; liquid-liquid interfaces; membranes; modified poly(vinyl chloride); solid-state electrodes.

\section{INTRODUCTION}

In recent years, the use of voltammetric methods as the read-out method of ion-selective membrane electrodes, traditionally interrogated under zero-current conditions, has created an important class of ion sensors. The application of an external stimulus to achieve ion extraction and detection is primarily useful to design sensors that exhibit improved operational reversibility and also set the stage for totalcharge-counting devices by coulometry. Specifically, such membrane electrodes can now be interrogated by cyclic voltammetry and amperometry [1], stripping voltammetry [2], normal-pulse voltammetry [3], pulsed chronopotentiometry [4,5], and thin-layer coulometry [6]. In contrast to potentiometric ion-selective electrodes, membranes applied in voltammetric techniques are typically chosen not to exhibit ion-exchanging properties in order to achieve a sample-membrane interface that can be effectively polarized by the electrochemical perturbation step. Upon an applied potential or cur-

*Pure Appl. Chem. 84, 1973-2063 (2012). A collection of invited papers based on presentations on the Novelty in Green Analytical Chemistry theme at the $14^{\text {th }}$ Asian Chemical Congress (14 ACC), Bangkok, Thailand, 5-8 September 2011.

¥Corresponding author 
rent, the ion of interest is extracted/transferred into the membrane phase, often with the help of a chemical receptor or ionophore in the membrane.

The charge-transfer process at the sample side of the membrane must be compensated at its inner side, which can be accomplished by the extraction of a counterion at that side [7], which becomes problematic for relatively thin membranes when the extracted analyte ion and its counterion may meet in the course of the experiment. More classically, a nonpolarizable interface can be chosen with an ion common to both phases [8]. In this case, however, there is a risk of this ion being lost from the membrane by ion exchange with the analyte ion, resulting in a change of the membrane composition with time.

Ideally, the second interface of the membrane should be stabilized by a solid-state ion to electron transduction element, resulting in an all-solid-state electrode. Conductive polymers applied between a metal electrode and the membrane were indeed used to this effect [2,9-12]. While this approach may give satisfactory results for low-current applications or thin-layer electrochemical systems, the limited redox capacity of the polymer limits the use of this approach to other systems [12]. For this reason, such conductive polymer layers are most attractive for use in potentiometric solid-state sensors [13].

Langmaier and Samec [14] demonstrated the application of redox-active species (decyl-ferrocene) freely dissolved in the membrane phase for the fabrication of all-solid-state voltammetric ion sensors. A relatively large redox capacity was observed, presumably because the quasi-liquid state of the membrane allows efficient diffusion of the redox-active species to and from the contacting metal electrode, hence facilitating the ion-transfer process at the sample-membrane interface. The main drawback of this approach is the likely leakage of the redox-active species from the membrane with time, owing to the hydrophilic nature of the oxidized (and hence electrically charged) ferrocinium species, thereby deteriorating the electrode properties.

We recently reported on poly(vinyl chloride) (PVC) membrane bulk chemically modified with ferrocene $(\mathrm{Fc})$ groups [15] to avoid this limitation. This modification was achieved by the so-called Huisgen cycloaddition ("click chemistry") between azide groups, bound onto PVC by partial substitution of the chlorine atoms [16], and alkyne functionalities on the Fc. The new material, FcPVC, was characterized with voltammetric transduction principles. FcPVC-based membranes were shown to have high reproducibility as well as the high redox capacity. We present here a study on the influence of the membrane composition on the electrochemical behavior of the sensor material, as well as an example of the utilization of such an FcPVC-based membrane in an analytical sensing protocol.

\section{EXPERIMENTAL}

\section{Reagents, materials, and equipment}

High-molecular-weight PVC, sodium azide, ethynylferrocene, L-ascorbic acid, copper sulfate pentahydrate, tetradodecylammonium tetrakis(4-chlorophenyl)borate (ETH 500), bis(2-ethylhexyl) sebacate (DOS), potassium hexacyanoferrate (III), potassium chloride, sodium perchlorate, sodium nitrate, sodium thiocyanate, anhydrous tetrahydrofuran (THF), and dimethylformamide (DMF) were purchased from Sigma Aldrich and used without further purification. Aqueous solutions were prepared by dissolving the appropriate salts in Milli-Q-purified distilled water. All electrochemical measurements were performed in a three-electrode configuration using a large-surface-area coiled platinum wire as a counter electrode, a double-junction $\mathrm{Ag} / \mathrm{AgCl} / \mathrm{sat}$. $\mathrm{KCl} / 1 \mathrm{M} \mathrm{LiOAc}$ as a reference electrode (MettlerToledo AG, Schwerzenbach, Switzerland) and the FcPVC-modified glassy carbon electrode, of $19.6 \mathrm{~mm}^{2}$ surface area, as a working electrode. Measurements were performed at ambient temperature with an Autolab PGSTAT128N (Metrohm Autolab, Utrecht, The Netherlands) using Nova 1.6 software. 


\section{Electrode preparation}

The membrane cocktail was prepared by dissolving $7.5 \mathrm{mg}$ of FcPVC, $37.5 \mathrm{mg}$ of DOS, and $5 \mathrm{mg}$ of ETH 500 in $1 \mathrm{~mL}$ of THF. DOS is a plasticizer commonly used as a solvent in PVC-based (liquid) membranes. The lipophilic electrolyte ETH 500 was added to locally provide counterions for the extracted analyte in the membrane and to keep the electrical resistance of the membrane low. Electrodes were prepared by drop-casting $40 \mu \mathrm{L}$ of cocktail and letting the THF evaporate overnight under ambient conditions.

\section{Electrochemical experiments}

Cyclic voltammetry experiments were performed with a $50 \mathrm{mV} \mathrm{s}^{-1}$ scan rate. The redox sensitivity of the membranes was tested in $0.1 \mathrm{M} \mathrm{KCl}$ solution with or without the presence of $1 \mathrm{mM} \mathrm{K}_{3} \mathrm{Fe}(\mathrm{CN})_{6}$. The potential was scanned between a 0.8 and $-0.5 \mathrm{~V}$ potential range, starting from $0.4 \mathrm{~V}$ and scanning toward negative potentials first.

The redox capacity of the FcPVC was tested in $10 \mathrm{mM} \mathrm{NaClO}_{4}$ solution by applying a $0.8 \mathrm{~V}$ potential for $30,60,120,300$, and $600 \mathrm{~s}$, each time followed by a discharge pulse at $0 \mathrm{~V}$ for $200 \mathrm{~s}$.

The chronopotentiometric calibration curves were performed by adding calculated volumes of $1 \mathrm{M}$ sodium acetate, chloride, nitrate, or perchlorate stock solutions to $50 \mathrm{ml}$ of deionized water to reach the desired final concentrations in steps of half an order of magnitude within a range of $\log a=-3$ to -1.5 . The protocol of the measurement was as follows: (1) a period of open-circuit potential (OCP) measurement, (2) an applied fixed current pulse of 1-s duration, (3) a zero-current pulse applied for $0.5 \mathrm{~s}$, and (4) the final regeneration pulse at the initially measured OCP was performed. The sequence was repeated 4 times for every 3,9 , and $27 \mu \mathrm{A}$ anion uptake pulse. The potential values at the end of the zero-current pulses were used as the analytical signal.

The stability of the signal generated by the FcPVC-modified electrode was examined by applying a 1 -s pulse at $0.5 \mathrm{~V}$, followed by a 20 -s regeneration period at the applied OCP using $10 \mathrm{mM}$ sodium perchlorate solution. The procedure was repeated 30 times.

\section{RESULTS AND DISCUSSION}

Preliminary studies on the electrochemical behavior of FcPVC-based ion-selective electrodes were reported in our previous paper, with a focus on the redox capacity during prolonged polarization experiments [15]. We previously explored the properties of electrodes modified with PVC that contained $3 \mathrm{~mol} \%$ of Fc groups (relative to the number of monomers of the polymer). We found its redox capacity superior over other redox-active materials used for solid-state electrodes [2]. The relative standard deviation of the measured signal was below $1 \%$ and, importantly, the material itself was not sensitive to redox-active species present in the sample solution. In this article we explore electrodes modified with different membrane compositions including Fc groups, plasticizer, and inert lipophilic salt content and explore their use as anion-sensing materials.

In view of realizing pulsed chronopotentiometric ion sensors and, eventually, thin-layer coulometric ion sensors, it is very much desired that the ion-selective electrodes possess the highest redoxcapacity possible. For this purpose, PVC containing 6 mol \% of Fc groups was chosen. This was the highest limit after considering the polymer solubility in the casting solvent, THF.

In a first attempt, electrodes overlaid with a drop-cast membrane of the most classical composition, with $1 / 3$ of the polymer and $2 / 3$ of plasticizer DOS, was chosen. Since the content of Fc groups in this membrane was higher than in our first report [15], it was necessary to evaluate the sensitivity of the electrode to redox-active species present in the sample solution (Fig. 1). As in previous work [15], the anionic ferrocyanide ion was chosen in solution, and the potential was first scanned toward cathodic values. This allows one to avoid anionic extraction of the marker into the ion-selective membrane that is 


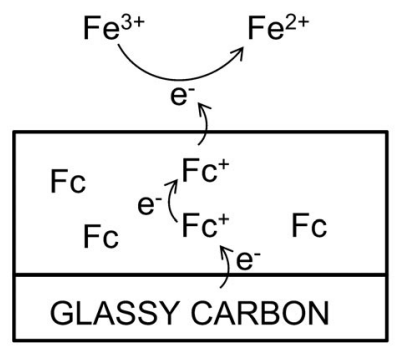

- POTENTIAL +
SAMPLE

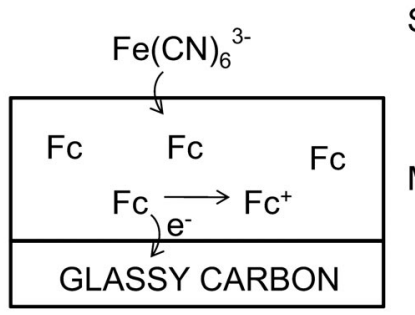

$\stackrel{-P O T E N T I A L}{\longrightarrow}+$

Fig. 1 Schematic representation of interactions of a redox-sensitive membrane with ferrocyanide anion in solution during a voltammetric scan toward cathodic potential values (left) and anodic potential values (right).

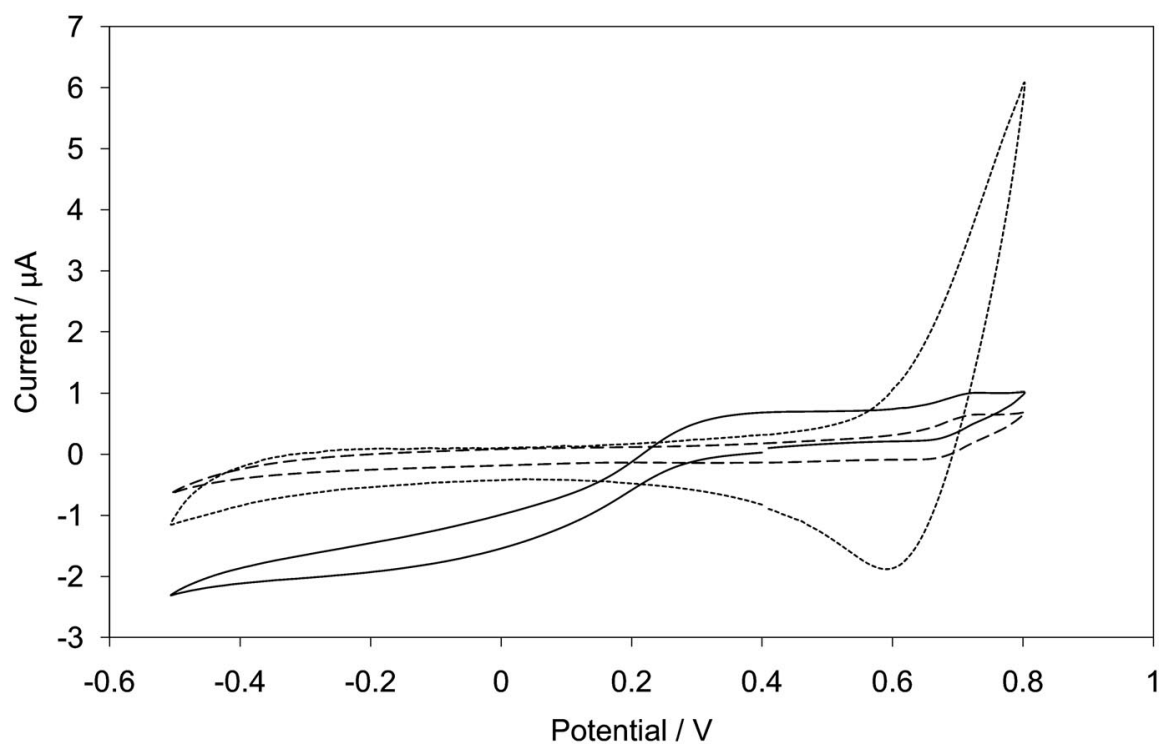

Fig. 2 Redox sensitivity of the 6 mol \% FcPVC-based membranes. Cyclic voltammograms were obtained for a membrane containing FcPVC and DOS in a $1: 2 \mathrm{wt}$ ratio recorded in a $1 \mathrm{mM} \mathrm{K}_{3} \mathrm{Fe}(\mathrm{CN})_{6}+0.1 \mathrm{M} \mathrm{KCl}$ solution (solid line), $0.1 \mathrm{M} \mathrm{KCl}$ only (dashed line) or in a $1 \mathrm{mM} \mathrm{K}_{3} \mathrm{Fe}(\mathrm{CN})_{6}+0.1 \mathrm{M} \mathrm{KCl}$ solution with an additional Fc-free membrane layer (dotted line).

expected to occur at anodic potentials. As can be seen in Fig. 2, a cathodic current of about $-2 \mu \mathrm{A}$ was observed below $-0.2 \mathrm{~V}$, which is ascribed to the reduction of ferrocyanide. This behavior suggests an efficient electron transport at the sample/membrane interface. At this concentration, the $\mathrm{Fc}$ groups in the membrane appear to serve not only as the electron donor but also as a conductor, suggesting either a sufficient mobility of these groups within the membrane phase or an efficient electron-hopping mechanism. However, no electron acceptor mechanism was expected owing to the lack of oxidized Fc form in the membrane. Therefore, when the electrode was scanned in the same potential range, but in a nonredox-active electrolyte, no reductive current related to the cation uptake was observed. Similarly, no reductive current was observed in the presence of ferrocyanide in the sample when the electrode was modified with an additional layer of Fc-free PVC/DOS/ETH 500 membrane (3:6:1 by mass). Here, the bound ferrocyanide solution ion was not able to undergo a reduction at the electrode surface owing to 
the lack of redox-active groups exposed to the solution. Alternatively, modifying the membrane composition by diluting the FcPVC with plasticizer (to a ratio of plasticizer to FcPVC of 5:1 by mass) significantly suppressed the aforementioned redox sensitivity. This suggests that below a critical concentration, Fc groups no longer participate in surface electrochemistry, presumably because the electron conduction of the bulk membrane is now sufficiently disrupted. Even the incorporation of ETH 500 to the membrane phase at $10 \mathrm{wt} \%$ did not return the surface redox activity.

The second most important parameter when considering the applicability of the redox-active material in solid-state voltammetric ion-selective electrodes is its redox capacity, which is much more important than with their all-solid contact potentiometric counterparts, where current flow is negligible. As in our previous work [15], we performed a controlled potential electrochemical doping step to study this quantity. The redox capacity of the $\mathrm{FcPVC}$ was tested in $10 \mathrm{mM} \mathrm{NaClO}_{4}$ solution by applying a $0.8 \mathrm{~V}$ potential for $30,60,120,300$, and $600 \mathrm{~s}$, each time followed by a discharge pulse at $0 \mathrm{~V}$ for $200 \mathrm{~s}$. For electrodes without ETH 500 when the membrane was doped for $10 \mathrm{~min}$, the charge was found as $12.7 \mu \mathrm{C} \mathrm{mm}^{-2}$, which is less than for the electrodes with the same surface area but based on $3 \mathrm{~mol} \%$ FcPVC $\left(26.5 \mu \mathrm{C} \mathrm{mm}^{-2}\right)$ studied previously [15] (see Fig. 3A). This can be explained by a slower charge transfer across the membrane bulk owing to the lack of lipophilic electrolyte, which results in a decreased doping rate. Moreover, the overall charge converted at the longest tested doping times is not equal to the maximum (thermodynamic) doping capacity of the membrane. Surprisingly, however, the doping capacity of electrodes based on 6 mol \% FCPVC and containing ETH $500\left(24 \mu \mathrm{C} \mathrm{mm}^{-2}\right)$ was still not larger than for the electrode reported before [15]. Since the concentration of the Fc groups in a membrane of the same composition is doubled, an increased redox capacity should be expected unless other processes (such as non-Faradaic charging) contribute to the overall signal, Alternatively, the $\mathrm{Fc}$ groups exhibit drastically different mobilities at the two different doping concentrations, which may dictate the turnover rates at the glassy carbon electrode. Interestingly, the electrochemical stripping signals shown on Fig. 3B did agree more closely with the doping levels. The stripping charge for $6 \mathrm{~mol} \%$ FcPVC-based electrode without ETH 500 after 10 min doping was found as $3.6 \mu \mathrm{C} \mathrm{mm}^{-2}$, the one containing lipophilic salt exhibited $12.7 \mu \mathrm{C} \mathrm{mm}^{-2}$, while the electrode based on $3 \mathrm{~mol} \%$ FcPVC showed only $1.5 \mu \mathrm{C} \mathrm{mm}^{-2}$. It is speculated here that the higher doping levels result in a lower mobility of the Fc groups, which will limit the current during the electrochemical doping process, but will remain more easily electrochemically accessible for the reverse reaction.

The stability of the measured signal was found to be very good. A cycle of a 1-s $500 \mathrm{mV}$ potentiostatic excitation pulse followed by a 20 -s OCP pulse was repeated 30 times. The current responses of the 500-mV pulses are shown in Fig. 4. The reproducibility of the 30 cycles was within $1 \%$ of relative standard deviation.

The suitability of the hybrid membrane and solid contact polymeric material for use in pulsed chronopotentiometric ion-selective electrodes was evaluated by use of a classical anion responsive system. Such an electrode is expected to respond to the Hofmeister selectivity sequence since no additional ionophore was added here. A four-pulse protocol, schematically shown in Fig. 5, was chosen in order to sample the cell potential at the end of a 1-s constant current pulse, and at the end of the immediately following 0.5-s zero-current pulse. This zero-current pulse is chosen to eliminate influences from the resistance and capacitance of the cell on the sensor signal. The role of the second pulse is the extraction of a defined amount of ions in the membrane, driven by the oxidation of the bound $\mathrm{Fc}$ at the back side of the membrane. The third pulse at zero current gives information akin to that of potentiometric sensors, but during the transient state that was achieved by the electrochemical perturbation of the second pulse. The anion-uptake pulse is applied under constant-current conditions, and should ideally result in a repeatable redox ratio of the Fc groups in the vicinity of the graphite electrode, giving a repeatable inner potential. Similarly, if no interference from other ions is observed and anion depletion is not relevant at the sample side, the anion-uptake pulse will induce the extraction of a repeatable amount of anions into the sample side of the membrane. In this ideal case, therefore, one expects an 

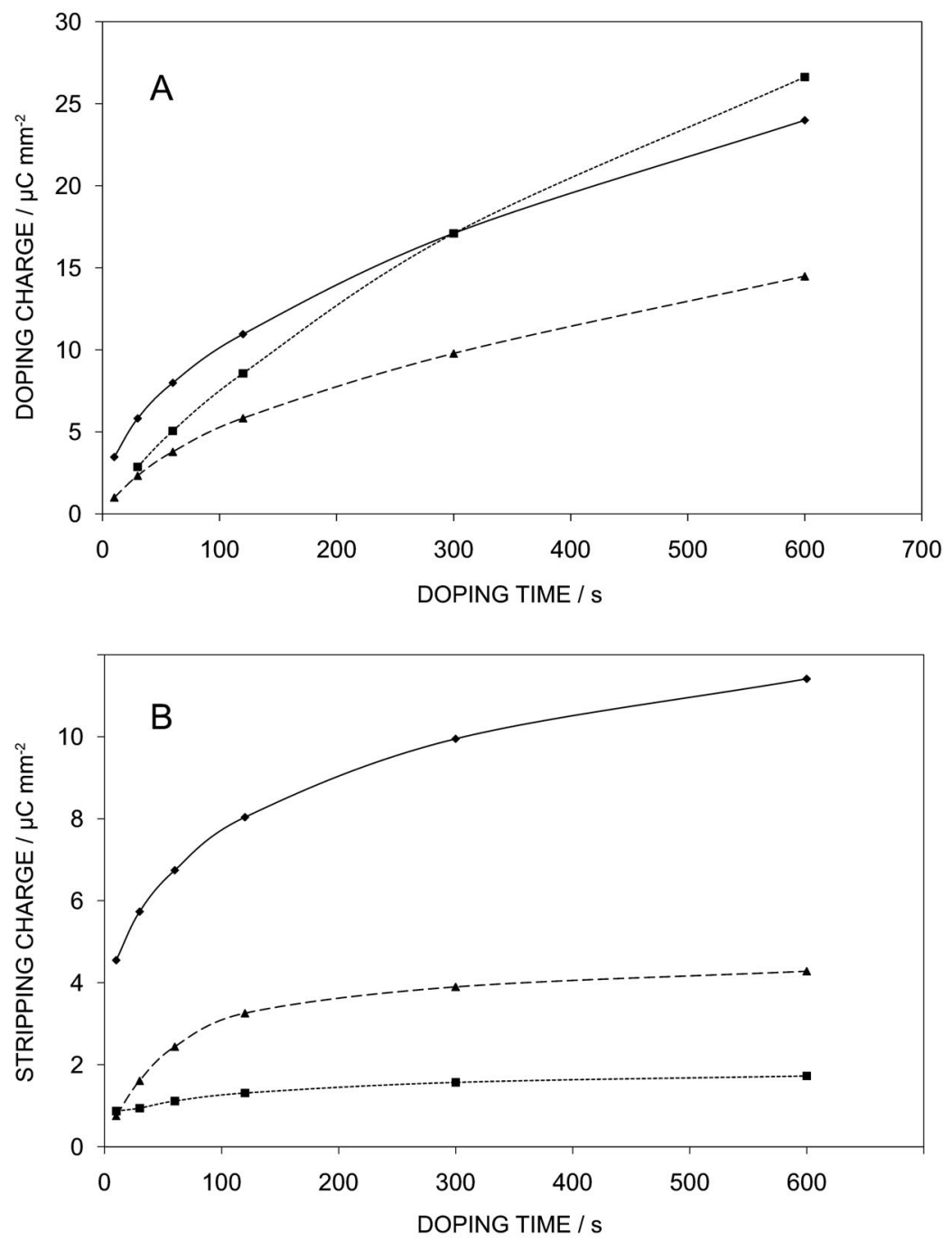

Fig. 3 (A) Doping and (B) stripping charge as a function of doping time for 6 mol \% FcPVC with (solid line) or without (dashed line) $10 \mathrm{wt} \%$ of ETH 500 in the membrane, and for $3 \mathrm{~mol} \%$ FcPVC with ETH 500 (dotted line). The membranes contained FcPVC and DOS in a 1:4 wt ratio. 


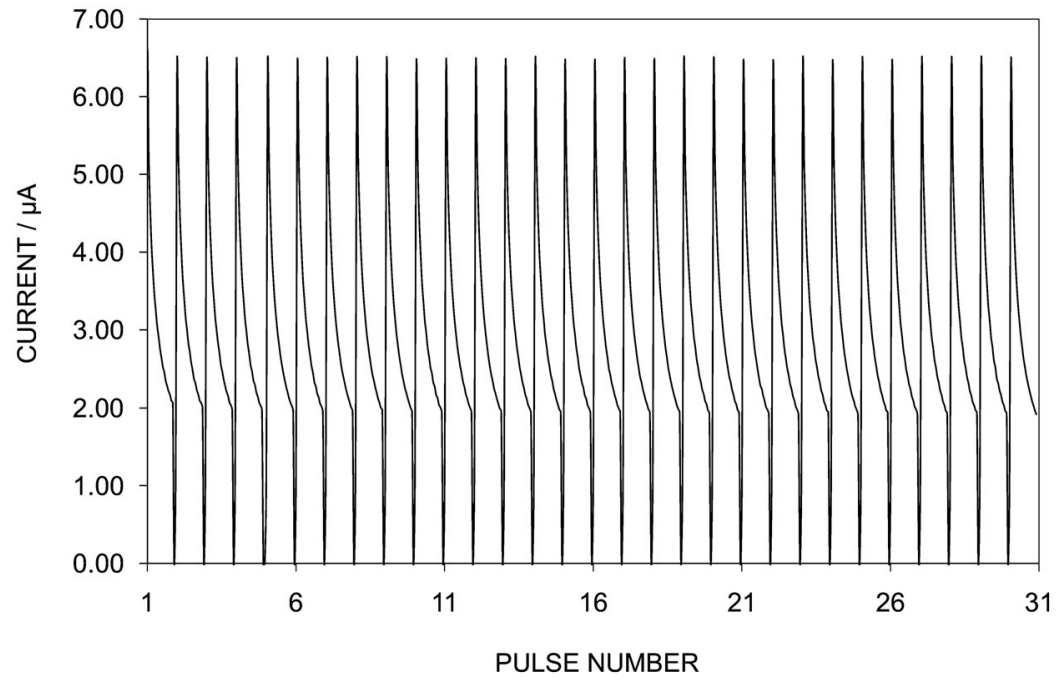

Fig. 4 Normal-pulse amperometry of an FcPVC-based membrane electrode immersed in $10 \mathrm{mM} \mathrm{NaClO}_{4}$ solution. Procedure consisted of 1 -s anion-uptake pulse at $0.5 \mathrm{~V}$ followed by 20 -s stripping pulse at $0.3 \mathrm{~V}$ (OCP, found before the experiment). The procedure was repeated 30 times. For clarity, only anion-uptake pulses are shown here.

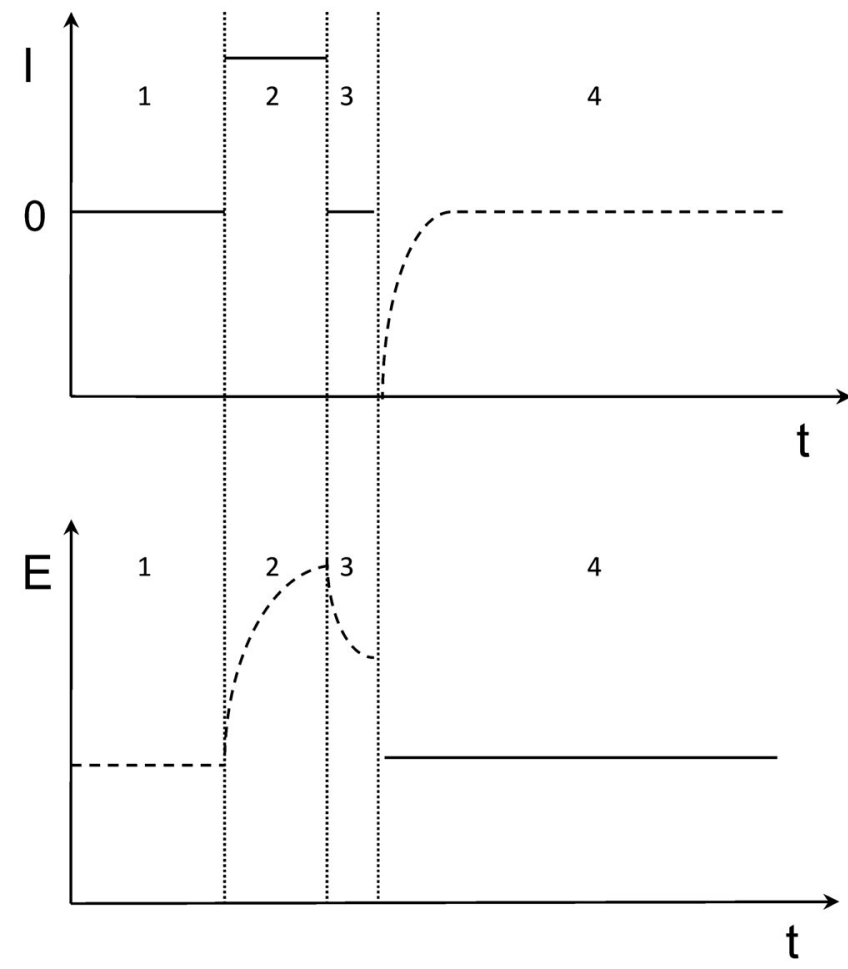

Fig. 5 Schematic representation of the potential and current changes with time during the galvanostatic/potentiostatic protocol used here, consisting of: (1) an OCP measurement, (2) a constant-current anion-uptake pulse, (3) a zero-current pulse, and (4) a constant potential anion-stripping pulse at the previously determined OCP value. 
apparently Nernstian response slope to the anion activity of pure electrolytes for potential readings acquired at a fixed time (at the end of each pulse).

Consequently, the procedure was applied to study the electrode behavior in various pure electrolytes. A 3- $\mu$ A current was applied for the first excitation pulse (data not shown). The electrode exhibited a high selectivity of perchlorate over nitrate of about three orders of magnitude (with a nitrate slope of $-48.6 \mathrm{mV}$ ) and over chloride of about five orders of magnitude (chloride slope of $-44.4 \mathrm{mV}$ ). The selectivity over acetate was, with three and a half orders of magnitude, lower than the ca. six orders expected, with a slope of $-45.2 \mathrm{mV}$. The electrode slopes found for the calibration curves were smaller than the Nernstian value. When the calibrations were repeated for higher excitation currents $(9 \mu \mathrm{A}$, data not shown) the slopes improved somewhat to $-49.3 \pm 4.1 \mathrm{mV}$ and the selectivity over acetate changed to five orders of magnitude. Only an even higher current of $27 \mu \mathrm{A}$ allowed us to obtain near-theoretical results with seven orders of magnitude selectivity over acetate and slopes of $-57.6 \pm 8.0 \mathrm{mV}$ (see Fig. 6A). The deviation of selectivity observed at mild excitation pulses perhaps arises from ion-binding properties of the linker used to attach the Fc group to the polymeric backbone. With increased current amplitude, the effect of selective interactions of the polymer with acetate ion may be overwhelmed by the increasing concentration of extracted ions.

The improvement of electrode slopes with increasing current density is not entirely clear. One expects the redox potential at the graphite electrode to be dependent on the ratio of oxidized and reduced $\mathrm{Fc}$ at that side of the membrane. This potential may be less reproducible at low current densities, when most Fc remains in the reduced form and transient processes result in more pronounced changes of the potential at the inner element. On the other hand, anion-exchanger membranes are known to exhibit similar characteristics in zero-current potentiometry. A higher ion-exchanger concentration in the membrane results in a more theoretical slope while low concentrations may more easily give slopes lower than Nernstian, especially for anions of limited lipophilicity [17]. The reason for this behavior may be related to additional transient transport phenomena or interferences, but have not clearly been elucidated in the literature.

After two weeks of storage in $10-\mathrm{mM} \mathrm{NaClO}_{4}$ solution, the calibration curves for perchlorate and acetate were repeated at $27 \mu \mathrm{A}$ applied-current amplitude (see Fig. 6B). The acetate calibration curve remained comparable to the one observed for the fresh electrodes shown in Fig. 6A, albeit with a smaller slope of $-45 \mathrm{mV}$. The calibration curve for perchlorate, on the other hand, exhibited a decrease in slope and a more important shift in the potential offset. These results suggest that there is no evidence for a strong poisoning of the membrane by perchlorate ions during the two-week storage period. This is consistent with results with liquid back-side-contacted pulstrodes, where poisoning by highly lipophilic ions was shown to be completely eliminated relative to classical potentiometric sensors where the extraction chemistry is not controlled instrumentally [18]. Nonetheless, the solid-contact-based membranes evaluated here are operationally still less robust than their liquid-contacted counterparts, and more work is needed to further stabilize their electrochemical response behavior for long-term use. 


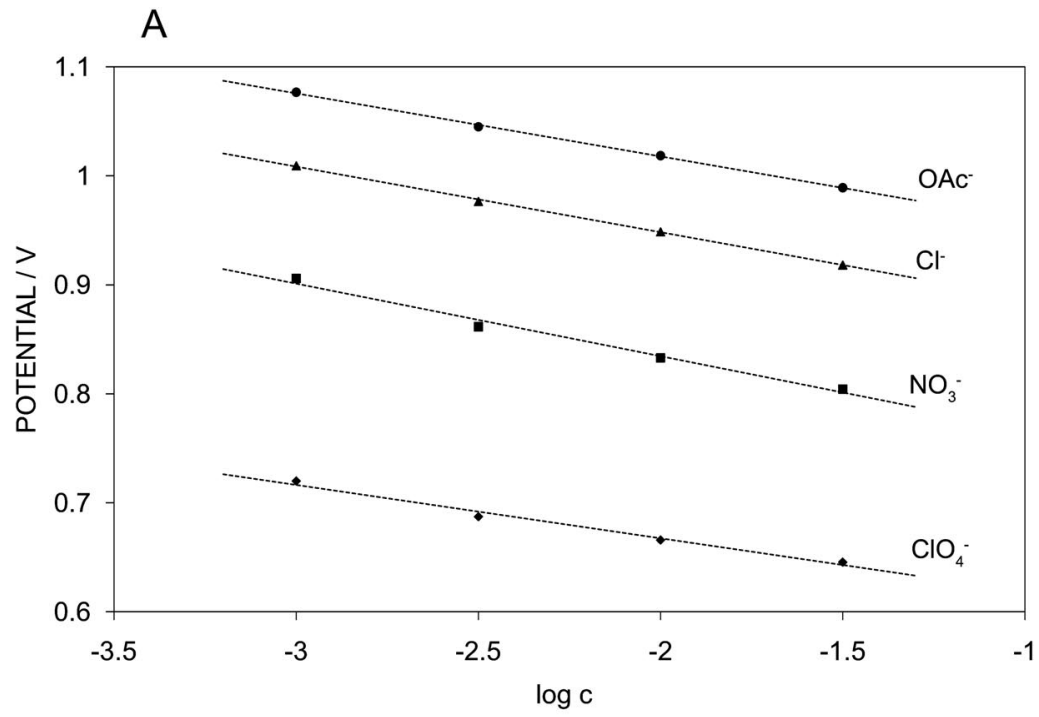

B

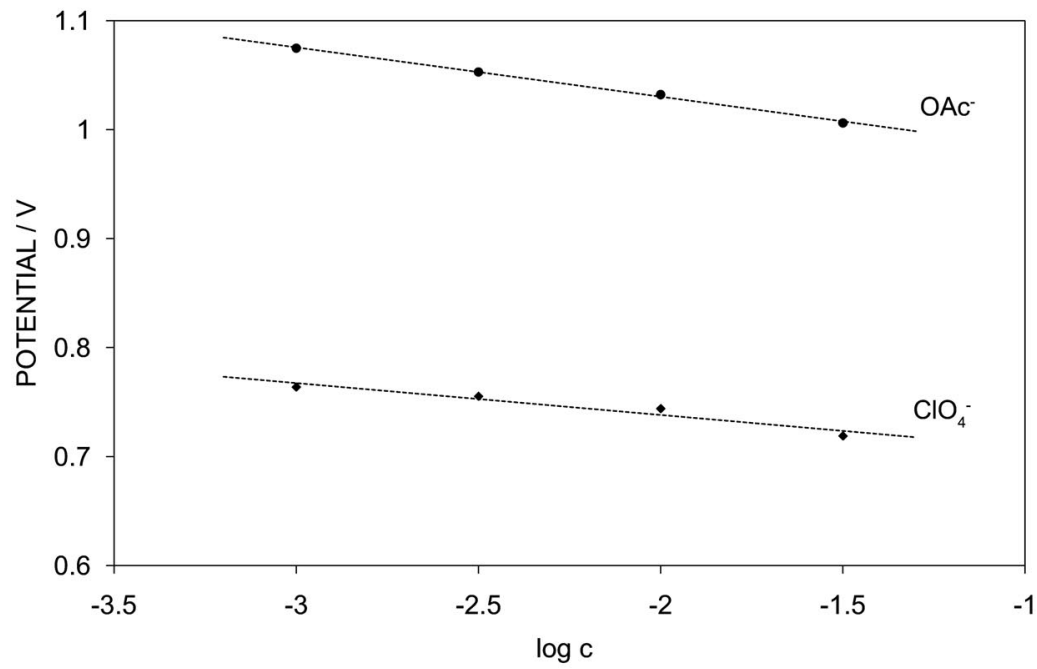

Fig. 6 (A) Calibration curves obtained for 4 different anions using the galvanostatic/potentiostatic protocol shown in Fig. 5 at $27 \mu \mathrm{A}$ excitation pulses, sampled at the end of the pulse. (B) Corresponding measurement at $27 \mu \mathrm{A}$ after two weeks of storage in $10 \mathrm{mM} \mathrm{NaClO}_{4}$ solution.

\section{CONCLUSIONS}

It was found that ion-selective membranes containing 2:1 plasticizer to FcPVC ratio suffer from sensitivity to redox-species present in the sample solution. Dilution of the FcPVC in the membrane down to 5:1 eliminated this effect. Chronoamperometric measurements showed, nonetheless, that the redox capacity of membranes based on $6 \mathrm{~mol} \% \mathrm{FcPVC}$, even after dilution with plasticizer, was found to be nearly an order of magnitude higher $\left(12.7 \mu \mathrm{C} \mathrm{mm}^{-2}\right)$ than for the membrane with $3 \mathrm{~mol} \% \mathrm{FcPVC}$ $\left(1.5 \mu \mathrm{C} \mathrm{mm}^{-2}\right)$ when the membranes were operated in an ion-stripping mode. The higher content of $\mathrm{Fc}$ groups halved the effect of capacitive current observed during the electrochemical ion-doping process, however. A previously reported multi-pulse procedure was used to demonstrate that such all-solid-state membrane function satisfactorily as pulsed chronopotentiometric sensors, with high current densities 
giving electrode slopes and selectivities closer to theoretical expectations. While storage in perchlorate solutions maintained the operational ion selectivity of the membrane, the long-term stability of the electrode may still need to be improved, perhaps by the use of a water-repelling intermediate layer between membrane and metal electrode.

\section{ACKNOWLEDGMENTS}

The authors thank the Swiss National Science Foundation and the Australian Research Council for supporting this research.

\section{REFERENCES}

1. Z. Samec, E. Samcova, H. H. Girault. Talanta 63, 21 (2004).

2. Y. Kim, S. Amemiya. Anal. Chem. 80, 6056 (2008).

3. S. Jadhav, A. J. Meir, E. Bakker. Electroanalysis 12, 1251 (2000).

4. A. Shvarev, E. Bakker. Anal. Chem. 75, 4541 (2003).

5. K. Gemene, E. Bakker. Anal. Chem. 80, 3743 (2008).

6. E. Grygolowicz-Pawlak, E. Bakker. Anal. Chem. 82, 4537 (2010).

7. S. Jadhav, E. Bakker. Anal. Chem. 71, 3657 (1999).

8. H. J. Lee, C. M. Pereira, A. n. F. Silva, H. H. Girault. Anal. Chem. 72, 5562 (2000).

9. A. Cadogan, Z. Gao, A. Lewenstam, A. Ivaska. Anal. Chem. 64, 2496 (1992).

10. A. Michalska, A. Hulanicki, A. Lewenstam. Microchem. J. 57, 59 (1997).

11. M. Vazquez, J. Bobacka, A. Ivaska, A. Lewenstam. Sens. Actuators B 82, 7 (2002).

12. P. Si, E. Bakker. Chem. Commun. 5260 (2009).

13. J. Bobacka, A. Ivaska, A. Lewenstam. Chem. Rev. 108, 329 (2008).

14. J. Langmaier, J. Olsak, E. Samcova, Z. Samec, A. Trojanek. Electroanalysis 18, 1329 (2006).

15. M. Pawlak, E. Grygolowicz-Pawlak, E. Bakker. Anal. Chem. 82, 6887 (2010).

16. V. V. Rostovtsev, L. G. Green, V. V. Fokin, K. B. Sharpless. Angew. Chem., Int. Ed. 41, 2596 (2002).

17. E. Pretsch, D. Wegmann, D. Ammann, A. Bezegh, O. Dinten, M. W. Laubli, W. E. Morf, U. Oesch, K. Sugahara, H. Weiss, W. Simon. In International Symposium on the Theory and Application of Ion-Selective Electrodes in Physiology and Medicine, pp. 11-16, Burg Rabenstein (1983).

18. K. L. Gemene, A. Shvarev, E. Bakker. Anal. Chim. Acta 583, 190 (2007). 